\title{
Nuclear Medicine Operations in the Times of COVID-19: Strategies, Precautions, and Experiences
}

\author{
Johannes Czernin ${ }^{1}$, Stefano Fanti ${ }^{2}$, Philipp T. Meyer ${ }^{3}$, Martin Allen-Auerbach ${ }^{1}$, Marcus Hacker ${ }^{4}$, Mike Sathekge ${ }^{5}$, \\ Rodney Hicks ${ }^{6}$, Andrew M. Scott ${ }^{7}$, Jun Hatazawa ${ }^{8}$, Mijin Yun ${ }^{9}$, Heiko Schöder ${ }^{10}$, Peter Bartenstein ${ }^{11}$, \\ and Ken Herrmann ${ }^{12}$
}

\begin{abstract}
${ }^{1}$ Ahmanson Translation Imaging Division, David Geffen School of Medicine at UCLA, Los Angeles, California; ${ }^{2}$ Department of Oncology, Division of Nuclear Medicine, University of Bologna, Bologna, Italy; ${ }^{3}$ Department of Nuclear Medicine, Medical Centre University of Freiburg, Faculty of Medicine, University of Freiburg, Freiburg, Germany; ${ }^{4}$ Department of Nuclear Medicine; Medical University of Vienna, Vienna General Hospital, Vienna, Austria; ${ }^{5}$ Nuclear Medicine Department, University of Pretoria and Steve Biko Academic Hospital, Pretoria, South Africa; ${ }^{6}$ Molecular Imaging and Therapeutic Nuclear Medicine, the Peter MacCallum Cancer Centre, Melbourne, Australia; ${ }^{7}$ Department of Molecular Imaging and Therapy, Austin Health, Melbourne, Australia; ${ }^{8}$ Department of Quantum Cancer Therapy, Research Centre for Nuclear Physics, Osaka University, Osaka, Japan; ${ }^{9}$ Department of Nuclear Medicine; Yonsei University Hospital System, Seoul, South Korea; ${ }^{10}$ Molecular Imaging and Therapy Service, Department of Radiology, Memorial Sloan Kettering Cancer Center, New York, New York; ${ }^{11}$ Department of Nuclear Medicine, University Hospital of Munich, LMU, Munich, Germany; and ${ }^{12}$ Department of Nuclear Medicine, Universitätsklinikum Essen, Essen, Germany
\end{abstract}

$\mathbf{T}$ he COVID-19 pandemic has now reached most countries and is straining health-care systems worldwide to their limits. Emergency room and intensive care staff are at increased risk for infection. Nobody knows the exact mortality rates associated with infections. However, given a likely very large number of infected patients, the number of fatalities will be high even if mortality rates are less than $1 \%$. As recent events in Europe indicate, hospitals may simply be overwhelmed by the high number of patients needing care.

Strategies to contain the spread of the virus are, therefore, essential. Outpatient hospital-based or free-standing imaging clinics are also exposed to asymptomatic carriers and patients with flulike symptoms. There are different ways to tackle this challenge, and this short article aims to provide you with an overview of different approaches supporting decision making. We briefly summarize strategies, precautions, and experiences from clinics in Europe (Fanti, Herrmann, Hacker, and Meyer), Australia (Hicks, Scott), Asia (Hatazawa, Yun), Africa (Sathekge), and the United States (Allen-Auerbach, Schöder) as implemented by local leadership. There will also be an example case recently discovered by the LMU Munich group (Bartenstein; Fig. 1).

\section{STEFANO FANTI, MD: DEPARTMENT OF ONCOLOGY, DIVISION OF NUCLEAR MEDICINE, UNIVERSITY OF BOLOGNA, BOLOGNA, ITALY}

We have started a triage for all patients accessing the building (nuclear medicine + radiation oncology). All patients are asked

Received Mar. 23, 2020; revision accepted Mar. 23, 2020.

For correspondence or reprints contact: Ken Herrmann, Department of Nuclear Medicine, Universitätsklinikum Essen, Hufelandstrasse 55, 45147 Essen, Germany.

E-mail: ken.herrmann@uk-essen.de

Published online Apr. 1, 2020.

Immediate Open Access: Creative Commons Attribution 4.0 International License (CC BY) allows users to share and adapt with attribution, excluding materials credited to previous publications. License: https://creativecommons. org/licenses/by/4.0/. Details: http://jnm.snmjournals.org/site/misc/permission.xhtml. COPYRIGHT ( 2020 by the Society of Nuclear Medicine and Molecular Imaging. DOI: 10.2967/jnumed.120.245738 for possible exposure, temperature is measured, and health status is evaluated before moving any further in the division. This appears to be working well for several reasons: (a) patients potentially positive are asked to go back home, thus reducing the risk of personnel exposure; (b) the personnel can limit the use of face masks, which are really lacking at present; (c) patients and personnel feel very reassured by it. We also have rescheduled all non-high-priority scans, namely routine control examinations: we have thus reduced the number of our imaging studies by $20 \%$. Given such reduction, 2 doctors of the staff, already experienced in ultrasound examinations, are ready to work in the COVID-19 Division to perform chest ultrasound to rapidly diagnose interstitial pneumonia. So far none of the staff has been infected, but one has been quarantined, living in a red area zone.

\section{KEN HERRMANN, MD, MBA: CHAIR, DEPARTMENT OF NUCLEAR MEDICINE, UNIVERSITÄTSKLINIKUM ESSEN, ESSEN, GERMANY}

We are anticipating what has happened in Italy and try to learn from this. For now, we have shut down all elective studies and therapies focusing solely on progressing tumor patients. The reduced program (reduction of thyroid outpatients by $95 \%$, SPECT by $70 \%$, PET by $60 \%$ ) allows us to implement 3 independent teams with only one being onsite. This will allow us to bring in new teams in case of infection or until a new "normal" has been defined. The current situation also teaches us that we need to integrate more internal medicine training into our programs.

\section{PHILIPP T. MEYER, MD, PHD: CHAIR, DEPARTMENT OF NUCLEAR MEDICINE, MEDICAL CENTRE - UNIVERSITY OF FREIBURG, FREIBURG, GERMANY}

To protect patients and personnel, patients are screened on arrival, which includes a brief questionnaire concerning contact persons and symptoms. In addition, body temperature is assessed. In case of suspicious findings (e.g., dry cough or elevated temperature), patients are liberally rescheduled to the next 1-2 d and 
advised to contact their general practitioner or our ER (if felt necessary). Patients with only mild coldlike or likely allergic symptoms (but no temperature increase) receive a face mask (as masks are limited, not every patient can get one). Examinations are focusing on short-term (2-3 mo.) progressive malignant and urgent cardiovascular diseases. We minimize exposure risk for the elderly by cancelling nonessential studies. We reduce the number of PET scans by $25 \%$, conventional scintigraphy (including SPECT/CT) by $50 \%$, and thyroid outpatient visits by $80-90 \%$ while maintaining radionuclide therapies in cancer patients. Concerning personnel, we established strictly separated teams with roughly half of our physicians working at home (also as backup) or as support in other departments. Other personnel (technicians, physicists, radiopharmacists) are strongly encouraged to stay at home (i.e., home office, taking care of children during school closure) whenever possible, especially those with risk factors (e.g., $>60 \mathrm{y}$-old, chronic diseases). Until now, we did not examine patients with known COVID-19 disease. Unlike other departments with regulations being less strict or issued later, we did not observe transmissions in our department up to now.

\section{MARTIN ALLEN-AUERBACH, MD: DIRECTOR OF NUCLEAR MEDICINE, DAVID GEFFEN SCHOOL OF MEDICINE AT UCLA, LOS ANGELES, CALIFORNIA}

We have implemented protective mechanisms on many levels. Staff continue to follow universal precautions and are instructed to practice frequent cleaning of high-touch surfaces (e.g., shared workstations and equipment). When staff experiences cough and fever they stay home. Staff with persistent symptoms and fever get tested as much as possible (testing capabilities remain woefully limited). We are attempting to have backup teams offsite who can take over if onsite staff falls ill. We are limiting imaging studies to those that are essential and urgent. Research studies are limited to a minimum. Front desk staff screens patients for flulike symptoms during reminder calls and on arrival in the clinic. Administrative staff is working remotely as much as possible.

While this section was being written, one staff member tested positive for SARS-CoV. We implemented the following protective measures: (a) minimized on-site staff; (b) reduced conventional nuclear medicine studies to emergency studies; (c) reduced the PET/CT volume to a level that can be managed by 2 technologists; (d) discontinued all research studies; (e) stayed in close communication with all team members to know when/if staff developed typical symptoms and fever; and (f) provided a list of patients who had contact with the infected staff member to the hospital command center.

We conduct daily morning leadership meetings including chief technologist, nursing, administration, and medical director to review the schedule, cancelling all nonessential studies; assessing staff census, symptoms, and test results; and sending out updated information to all our staff.

\section{MARCUS HACKER, MD: DIRECTOR OF NUCLEAR MEDICINE, MEDICAL UNIVERSITY OF VIENNA, VIENNA GENERAL HOSPITAL, VIENNA, AUSTRIA}

Austria has shut down public life, meaning that people should not leave their houses; schools and universities as well as public institutions, stores, and restaurants are closed. These activities aim to slow down the spread of the virus due to the limited intensive care beds in Austria, which would be fully occupied within a short time frame and, thus, increase local mortality rates. The Vienna General Hospital has administrated strict entry regulations for both patients and staff. Patients with symptoms or fever are guided to a specialized center for triage and CT scans. Personal staff can only enter the building after showing their hospital identification. in nuclear medicine, we cancelled every elective diagnostic procedure (such as thyroid controls or PET/MRI) and therapy (e.g., radioiodine treatment). Emergency scans or those examinations that are guiding treatment decisions are still performed. Personal staff is now organized in 2 teams, with weekly rotations from home office to clinic, so that a complete shutdown in the case of emerging infections can be avoided. Personnel not needed in the nuclear medicine department help compassionately in other divisions.

\section{MIKE SATHEKGE, MD, PHD: HEAD OF NUCLEAR MEDICINE DEPARTMENT, UNIVERSITY OF PRETORIA AND STEVE BIKO ACADEMIC HOSPITAL, PRETORIA, SOUTH AFRICA}

Our nuclear medicine staff have been instructed to have refresher courses on infection control management and basic emergency management. In line with the university hospital policy, we have rearranged our front desk to enable screening of all patients coming to the department. For patients with suspected COVID-19 we cancel the procedure and refer them to the hospital dedicated COVID-19 team, which is led by an infectious disease specialist. Together with the referring physicians, we have reduced elective cases, based on the priority of the scheduled treatment. Some of our follow-up patients who are stable have been postponed and consultation done via telephone. We have deferred our multidisciplinary meetings and replaced them by telecommunications between a few deciding physicians. We have asked outpatients, where possible, not to come with accompanying people.

In Africa, we expect the COVID-19 pandemic to be worse as our winter is approaching, more so that we have relatively weak health systems, overcrowded houses, and a large population that is immunocompromised.

\section{RODNEY HICKS, MD: DIRECTOR, MOLECULAR IMAGING AND THERAPEUTIC NUCLEAR MEDICINE, THE PETER MACCALLUM CANCER CENTRE, MELBOURNE, AUSTRALIA}

As Australia's only dedicated cancer hospital, we have a population of patients who are uniquely at risk of infection, both due to their immune status and the need to attend hospital frequently for treatment. A particular challenge is dealing with patients with fever, cough, or shortness of breath since these are common manifestations of opportunistic infection, pneumonitis from radiation or immune checkpoint inhibitors and thromboembolism in our population as well as being features of COVID-19. It appears that the imaging features of lung involvement in the COVID-19 setting mimic those of other viral infections in the neutropenic patient and can also have features similar to immune-associated pneumonitis due to immune-checkpoint inhibitor therapy. Accordingly, we balance caring for the real and present danger that poor management of their underlying oncologic condition poses against the risk of exposing our staff and other patients to viral infection. We are managing this by screening all patients arriving at the department for potential risks of COVID-19 and isolating those deemed at increased risk for medical assessment of the need for their planned scan. If medically urgent, we put in place stringent infection-control measures around performance of the scan, just as we do with 
other communicable diseases. At this time, we are also acutely aware of both the physical and the mental health of our staff, particularly our receptionists and technologists who are at the front-line of patient interaction. We are stressing the need for kindness and consideration to each other, supporting those who have family members or who themselves need to be confined to home and establishing teams who work together to minimize contacts in the event that a team-member becomes infected. Multidisciplinary meetings are core to our management model but have gone "virtual," and wherever possible we are doing telehealth consultations, especially for our radionuclide therapy patients in surveillance. Finally, our profession is one of the truly global communities, and as the world closes its borders and becomes more nationalistic and xenophobic, we have a responsibility to support each other and to advocate for humanist principles of care and compassion for all.

\section{ANDREW SCOTT, MD: DIRECTOR, DEPARTMENT OF MOLECULAR IMAGING AND THERAPY, AUSTIN HEALTH, MELBOURNE, AUSTRALIA}

We have implemented active measures to identify possible COVID-19 patients arriving to our Department and have implemented appropriate procedures for infection control. Teams have been established for all areas (administration/reception, technologists, physicians, scientists) to minimize exposure and ensure backup in the event of staff being forced to self-isolate. Our clinical service has seen reductions in nonurgent scans, while inpatient and urgent outpatient studies (particularly oncology) are continuing at similar levels. Clinical multidisciplinary meetings are being conducted by videoconference, and patient consultations are being conducted by teleconference where possible. Teaching and training programs have been halted at this time. A hold on recruitment to clinical trials has also been implemented in our institution and will have an impact on our research programs. Although we are fortunate in Australia to have a local supply of many radioisotopes, and therefore are not likely to be affected by shortages as may occur in other countries, we are reliant on overseas supply for many kits and reagents and the shutdown of borders and airline flights may be problematic in the coming months. Strategies to address possible access issues for radiopharmaceuticals are being addressed by our national nuclear medicine leadership in a rapidly evolving COVID-19 landscape.

\section{JUN HATAZAWA, MD, PHD: DEPARTMENT OF QUANTUM CANCER THERAPY, RESEARCH CENTRE FOR NUCLEAR PHYSICS, OSAKA UNIVERSITY, OSAKA, JAPAN}

The first patient with COVID-19 infection was reported on January 22, 2020, in Japan. Since then, we have faced many challenges. Nuclear medicine is being practiced according to the general medical guideline during the outbreak of COVID-19. First, reconsider the necessity of the examination at this occasion; second, avoid crowding among patients and medical staff, and discard infectious medical waste separately. The number of imaging studies has decreased slightly this month. When suspected or confirmed patients are examined, medical staff need the highest protection level by wearing disposal caps, glasses, medical masks, disposal protective clothing/impermeable isolation clothing, hand gloves, and shoe covers. Use of these protection materials was proven to be effective during the relief operation on the cruiser "Diamond Princess," resulting no infected rescue personnel. Nuclear medicine depends on a global supply chain of radionuclides and radiopharmaceuticals. Supply of radionuclides remains stable, but will be a serious concern when nuclear reactor production outside Asia is restricted and intercontinental transport by aircraft is limited. We test COVID-19 viruses, and COVID-19 viruses test the societies of human beings.

\section{MIJIN YUN, MD: DEPARTMENT OF NUCLEAR MEDICINE, YONSEI UNIVERSITY HOSPITAL SYSTEM, SEOUL, SOUTH KOREA}

In South Korea, nationwide screening programs have significantly contributed to limiting the spread of the COVID-19. Anyone with respiratory symptoms, instead of directly visiting outpatient clinics or emergency rooms, are encouraged to go to well-organized screening centers including drive-through centers and send their samples to government-certified test centers, which have the capability of performing 15,000 tests per day. After testing, test-positive patients would be triaged according to the extent of symptoms, age, underlying diseases, risk factors, and the like into designated hospitals or specialized care centers under the supervision of infectious disease specialists for isolation. And by contact tracing, it is recommended for people in close contact with those who are infected to stay home for $2 \mathrm{wk}$ and get a test as needed. As of now, approximately 290,000 people have been tested and a total of 8,652 infections and 94 mortalities were found, with a sharply declining trend of new cases.

At a hospital level, there are outside screening spots for those with respiratory symptoms or possible exposure or from certain regions of outbreak. The access to enter the building is limited to control the flow of people, and everyone at the gate is screened for possible exposure. Visitors and hospital staff members should wear facial masks and frequently use hand sanitizer. In addition, there is a notification in the hospital electronic medical record system, to help medical personnel recognize patients with possible COVID-19 symptoms or those from regions with high numbers of infected patients. Patients with respiratory symptoms who need nuclear medicine examinations are asked to come at the end of day, and after the study the designated area is specially cleaned by quarantine people in addition to a routine cleaning program. A specialized device that can have a patient under negative pressure has been used for infected patients for their chest CT scans. At the same time, we call the scheduled patients asking their health status and whether they want to reschedule the examinations at their convenience. Due to the nature of a tertiary medical hospital, the reduction in the number of studies was mild. Therefore, multilevel preventive and protective strategies including rapid and efficient screening programs for the detection of coronavirus, contact tracing, proper screening, control the flow of high-risk people, personal hygiene, social distancing, strict adherence to the hospital rules, and installment of a quarantine system would add up to combat the COVID-19.

\section{HEIKO SCHÖDER, MD, MBA: CHIEF, MOLECULAR IMAGING AND THERAPY SERVICE, DEPARTMENT OF RADIOLOGY, MEMORIAL SLOAN KETTERING CANCER CENTER (MSKCC), NEW YORK, NEW YORK}

MSKCC started imposing travel restrictions (no attendance of any outside meeting, even if locally in New York) about 2 wk ago (early March, 2020), and more recently has implemented teleconferencing for all internal meetings and tumor boards. A departmental 
leadership committee makes decisions regarding patient care and staffing levels in the department. Each radiology division has in place a 4-tier plan in which examinations are reduced in a stepwise fashion. For nuclear medicine, the first level includes delaying most radioiodine treatments and cancelling certain conventional nuclear medicine studies and FDG PET scans. In the final tier, only true emergency studies will be offered. In patients undergoing nuclear medicine pulmonary embolism studies, we have eliminated the ventilation portion. Coincidentally, we are noticing 2 trends: (a) patients are "self-selecting," that is, deciding not to undergo imaging studies they consider nonessential and (b) all other departments are reducing their number of elective procedures (e.g., surgeries, radiation treatments), causing less demand for related PET scans. Regarding our therapy operations, we are also converting all initial and follow-up clinical consults to telemedicine, thus reducing direct patient contact. All laboratory research endeavors have already been shut down; researchers are working from home. Medical physicists have implemented a rotation system; only one third of them are on premises on any given day to assist with hardware and software issues. Physicians assigned to research or administrative duties are asked to conduct these from home.

As we are operating in a given administrative, geographic, and political context, our operations are influenced by decisions made by hospital administrators and elected officials. At MSKCC, a hospital leadership group oversees crisis management and issues policies and guidelines. Critical decisions are communicated via mass email to all staff several times per day, and an internal website has been set up to address questions and concerns by patients and staff. Despite these efforts, much uncertainty remains as we are dealing with an "invisible enemy." For instance, whereas we (like other institutions) screen for symptomatic patients and redirect them to the hospital's urgent care center before entering our clinic, we need to recognize that this only addresses the tip of the iceberg: it is increasingly clear that we currently do not have the means to recognize and isolate infected individuals (viral carriers who can spread the disease) in real time, before they become symptomatic. Moreover, while the number of COVID-19 patients is increasing, nationwide we are still facing shortages in N95 protective masks. Staff need to be prepared for assignments outside their normal clinical work environment.

\section{SUMMARY}

One of the biggest challenges in daily clinical routine will be to think of COVID-19 findings, especially in patients not suspected of being COVID-19-positive. The LMU group recently discovered such a case (Fig. 1).

The variety of approaches applied in different departments and on 5 different continents hopefully provides guidance, ideas, and concepts to successfully combat the COVID-19 pandemia. The apparent common denominators are (a) triage patients, (b) reduce

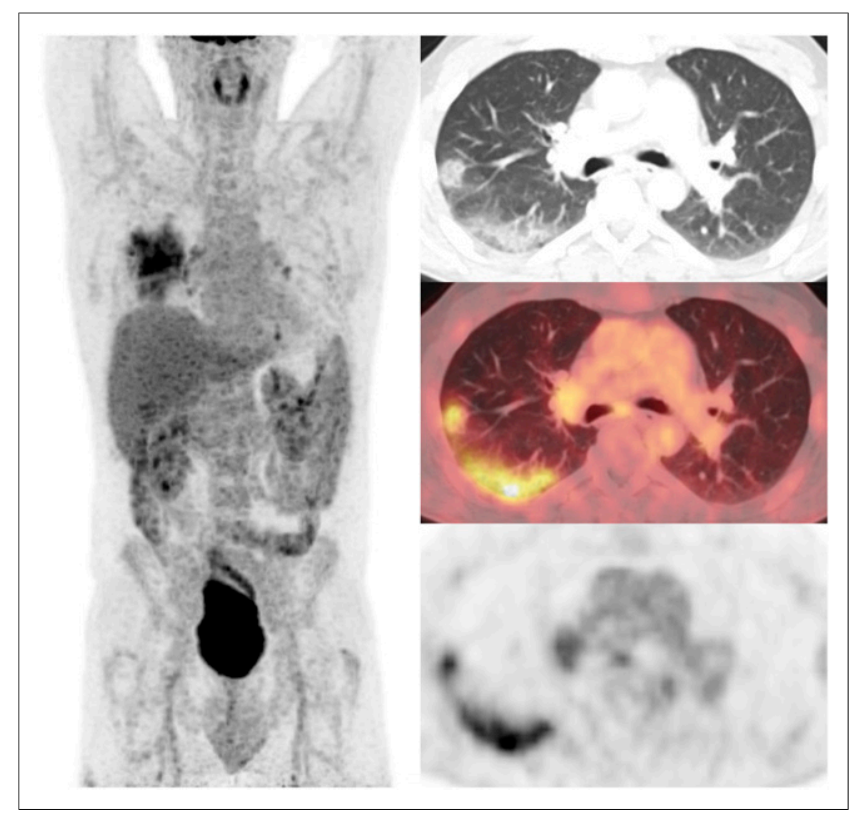

FIGURE 1. ${ }^{18} \mathrm{~F}-\mathrm{FDG}$ PET/CT in a 53-y-old patient with NET of the pancreas (G3 tumor) referred for restaging. At the time of the PET scan, the patient was completely asymptomatic. The current ${ }^{18} \mathrm{~F}$-FDG PET/CT displays a new hypermetabolic area in the right upper and lower lobe (SUV max $_{\text {a }} 5.5$ ), in topographic correlation to predominantly in the periphery/subpleurally located ground glass opacities with beginning, partly round shaped consolidations consistent with atypical inflammation. Later COVID-19 infection was confirmed.

elective studies, (c) increase hygiene, and (d) establish rotations to put in place back-up teams. We are all facing the beginning of COVID-19. None of us has the "best" solutions. This is new to all of us. Let us keep communicating and learning from each other.

\section{TAKE HOME MESSAGES}

- Screen patients on arrival.

- Use increased hygiene, including face masks (if available), personal protection device (PPD) (if confirmed).

- Perform no elective scans or procedures.

- Implement, if possible, separate teams.

- Secure on-site radiotracer production (if appropriate and feasible).

- Use social distancing (at work and in private life).

- Use telephone or video conferencing whenever possible.

\section{DISCLOSURE}

No potential conflict of interest relevant to this article was reported. 